\title{
ORIGINAL
}

\section{CARACTERISTICAS DE LAS PERSONAS QUE ACUDEN A LAS CONSULTAS DE DEMANDA DEL CENTRO DE SALUD SIN CITACIÓN PREVIA}

\author{
Carlos Isanta Pomar (1), Pilar Rivera Torres (2), Marta Pedraja Iglesias (2) y Natalia Giménez \\ Blasco (1). \\ (1) Centro de Salud Perpetuo Socorro. Huesca. \\ (2) Facultad de Ciencias Económicas y Empresariales. Departamento de Economía y Dirección de Empresas. Zaragoza.
}

\section{RESUMEN}

Fundamento: El tiempo de espera para acceder a la consulta está considerado un indicador de satisfacción. Las visitas no urgentes, realizadas sin cita previa, provocan un incremento del tiempo de espera de los pacientes citados, con la consiguiente insatisfacción de los mismos. El objetivo del estudio persigue la valoración cuantitativa de este tipo de visitas y conocer las características de las mismas, para introducir las medidas destinadas a su corrección y racionalización.

Métodos: Estudio descriptivo. Se realizó un registro que recogiera las características de los pacientes que originan las consultas sin cita previa, como li edad y el sexo y las relativas a la consulta: motivo, modalidad, jornada laboral y si era realizada de forma directa o indirecta.

Resultados: El 14,19\% de consultas a demanda no urgentes son sin citación previa. Con el análisis factorial de correspondencias múltiple se identifican dos perfiles de consultas: las directas, solicitadas por pacientes jóvenes, por enfermedad o tareas burocráticas, al final de la consulta de la mañana, estando ellos presentes, y las consultas indirectas, ocasionadas durante las consultas con cita previa por pacientes que solicitan recetas.

Conclusiones: Hay un elevado número de visitas sin cita previa. Son precisas medidas que puedan conducir a la utilización del sistema de organización de las consultas y a la mejora de aquellas situaciones que impliquen una falta de accesibilidad, con la finalidad última de mejorar la satisfacción de los usuarios de los servicios sanitarios públicos.

Palabras clave: Cita previa. Utilización de servicios sanitarios. Análisis factorial de correspondencia.

Correspondencia:

Carlos Isanta Pomar

Parque de Roma B-1, 10 B

50010-Zaragoza.

\section{ABSTRACT}

\section{Characteristis of those Individuals Requesting to be seen by a Physician wit- hout First Having Made an Appointment Ahead of Time}

Background: Waiting time to see a physician is considered to be an indicator of user satisfaction. Non-emergency visits made without an appointment ahead of time result in longer waiting times for the patients who have appointments, resulting in the consequent insatisfaction thereof. The purpose of this study is aimed at conducting a quantitative assessment of this type of visits and at ascertaining the characteristics thereof for the purpose of putting measures into practice for the correction and rationalization thereof.

Methods: Descriptive study. A record was made which would gather the characteristics of those patients seeing physicians without having made an appointment, such as age, gender and the characteristics related to the visit (reason, modality, working hours and whether made directly or indirectly).

Results: No appointment had been made ahead of time for $14.19 \%$ of all non-emergency patient-requested visits. Based on the factorial analysis of multiple correlations, two visit profiles are identified, that is, the direct visits requested by young patients due to illness or red tape at the end of the physician's morning hours, said young patients being present, and the indirect visits during the visits for which appointments have been made ahead of time by patients requesting prescriptions.

Conclusions: There are a large number of visits for which no appointment has been made ahead of time. Measures must be taken for the purpose of channeling the use of the appointment organization system and of improving those situations involving a lack of accessibility for the end purpose of improving the degree of satisfaction of those using the public health care services.

Key words: Appointment. Use of health care services. Factorial correlation analysis. 


\section{INTRODUCCION}

El tiempo que esperan los pacientes para acceder a la consulta del médico forma parte de una de las dimensiones de la accesibilidad a los servicios sanitarios, estando estrechamente relacionada con el grado de satisfacción de los usuarios ${ }^{1}$.

La consulta a demanda en el centro de salud, es una modalidad que, si bien surge a iniciativa del paciente o de forma concertada por el propio médico, precisa de una citación previa, estando cnmarcada en un horario preestablecido y por tanto conocido ${ }^{2}$. Dentro de las actividades asistenciales espontáneas se producen un número de visitas que, sin ser urgentes, son solicitadas por el propio paciente, directamente al médico, en el centro de salud y sin previa citación. Estas consultas originan una distorsión, tanto en el quehacer del profesional sanitario ${ }^{3}$, como en el resto de pacientes que acuden a la consulta a demanda con citación, ya que generan, entre otros inconvenientes, un incremento del tiempo de espera.

El tiempo de espera para acceder a las consultas ha sido considerado tradicionalmente como un indicador de satisfacción de los usuarios ${ }^{7,8}$ y como tal viene siendo recogido por el Insalud ${ }^{9}$ y a su vez trasladado a los EAP como un objetivo en los sucesivos contratos de gestión, considerando que para el año 2000 el porcentaje de usuarios con un tiempo de espera menor o igual a 15 minutos, debería ser superior al $70 \%$, así como el porcentaje de usuarios con tiempos de espera superiores a 30 minutos sea inferior al $10 \%$. La introducción de los programas de cita previa ha supuesto una mejora en los tiempos de espera ${ }^{10}$, sin embargo, traslada la referencia de un número de orden a una referencia horaria, lo que exige un cierto grado de disciplina, fundamentalmente por parte del médico, para el cumplimiento de los horarios establecidos ${ }^{11}$. Las visitas sin cita previa realizadas durante el tiempo de consulta, podrían neutralizar, en parte, la mejora conseguida con la cita previa, produciéndose un aumento de los tiempos de espera y la consiguiente insatisfacción de los usuarios. La utilización de los tiempos previo y posterior a la consulta constituye un problema diferenciado, ya que dificulta la realización de otras actividades asistenciales y no asistenciales y conlleva una desestructuración de la jornada laboral.

El objetivo del estudio consiste en la cuantificación de las visitas realizadas sin citación previa, así como en el conocimiento de las características de los pacientes que originan dichas visitas y el momento de la consulta en el que solicitan ser atendidos, lo cual nos puede permitir la introducción de medidas destinadas a la corrección y racionalización de las mismas.

\section{SUJETOS Y METODOS}

Se trata de un estudio descriptivo prospectivo llevado a cabo en el centro de salud Perpetuo Socorro de Huesca, que atiende a una población de 12.310 habitantes. En marzo de 1991 se puso en funcionamiento el Sistema de Citación Previa (SCP) que ya en 1989 fue introducido para todo el territorio Insalud, basándose en las experiencias previas del Servicio Vasco de Salud ${ }^{4}$. En el organigrama de funcionamiento del centro, las urgencias que surgen en horario de 9 a 14,30 horas son atendidas por todos los profesionales, en turnos rotatorios, quienes atienden, además, a todos los pacientes que, sin solicitar consulta urgente, no consideran oportuno demorar su visita al día siguiente, al no poder ser citados ya en la consulta de su médico o, incluso, no quieren ser citados en la consulta de su propio médico aunque por el horario fuera posible. Desde el 13 de junio de 1995 se instaló «la jornada deslizante», en la que cada uno de los médicos, un día a la semana, traslada su jornada laboral a la tarde, en horario de 14 a 20 horas. El estudio se llevó a cabo en una de las siete consultas de medicina general del equipo de atención primaria, con horario de consulta.a demanda de 9 a 11,45 horas todos los días de la semana, salvo los martes, en los que la consulta es por la tarde. La elección de dicha consulta no se realizó de forma aleatoria, sino que se eligió la consulta de uno de los médicos participantes del estudio ya que en un estudio piloto 
realizado anteriormente, se detectó una falta de anotación de los pacientes sin cita por el resto de profesionales, lo que alteraría la validez interna del estudio, sin que con ello, creemos, se pudiera ver afectada la validez externa del mismo.

En el estudio se distinguen dos períodos diferenciados, uno que va desde el 1 de enero de 1996 hasta el 31 de diciembre de 1998, durante el cual se cuantificó el número total de visitas, para valorar lo que hasta entonces había sido sólo una intuición. Para ello, se anotaron diariamente en la hoja de citación de consulta todas aquellas visitas realizadas sin cita previa. En un segundo periodo, que nos servirá para la valoración del perfil de la consulta, se estudiaron todas las visitas sin citación previa desde el 1 de octubre de 1997 al 31 de enero de 1998, para lo que se diseñó una hoja de recogida de datos, en la que se incluían las siguientes variables (tabla 1): A) Edad. B) Sexo. C) Motivo de la consulta: como motivo de consulta se consignaron los siguientes: a) Por enfermedad. b) Por recetas c) Por informes: incluyendo la solicitud de informes clínicos como tales, informes para la solicitud de balnearios, Inserso u otros motivos, los certificados médicos, los volantes de oxigenote-

Tabla 1

Definición de las variables analizadas

\begin{tabular}{|c|c|}
\hline VARIABLE & CATEGORIAS \\
\hline EDAD & EDAD DE LOS PACIENTES \\
\hline SEXO & $\begin{array}{l}\text { - HOMBRE } \\
\text { - MUJER }\end{array}$ \\
\hline MOTIVO DE LA CONSULTA & $\begin{array}{l}\text { - ENFERMEDAD } \\
\text { - RECETAS } \\
\text { - INFORMES: incluye la solicitud de informes clínicos como tales, } \\
\text { informes para solicitud de balnearios, INSERSO u otros motivos, cer- } \\
\text { tificados médicos, volantes de oxigenoterapia, volantes para ambu- } \\
\text { lancias y volantes de ordenes de inyectables. } \\
\text { - SOLICITUD DE IT O PARTES DE CONFIRMACION } \\
\text { - SOLICITUD DERIVACION A OTROS ESPECIALISTAS } \\
\text { - SOLICITUD ANALITICAS } \\
\text { - RECOGIDA DE RESULTADOS }\end{array}$ \\
\hline MODALIDAD DE LA CONSULTA & $\begin{array}{l}\text { - ENTRE PACIENTES: cuando la consulta era solicitada al introducir- } \\
\text { se el paciente entre otros pacientes citados } \\
\text { - ACOMPAÑANDO AL PACIENTE: cuando para la realización de la } \\
\text { consulta se aprovechaba la consulta realizada por un paciente citado } \\
\text { - AL FINAL DE LA CONSULTA: visitas realizada a pacientes que } \\
\text { esperan la finalización de la consulta o cualquier otro momento des- } \\
\text { pués de ya finalizada la consulta a demanda } \\
\text { - ANTES DE INICIAR LA CONSULTA A DEMANDA }\end{array}$ \\
\hline TIPO DE CONSULTA & $\begin{array}{l}\text { - DIRECTA } \\
\text { - INDIRECTA: aquella en la que el paciente no está presente }\end{array}$ \\
\hline MOMENTO DE CONSULTA & $\begin{array}{l}\text { - MAÑANA } \\
\text { - TARDE: coincidiendo con la jornada deslizante }\end{array}$ \\
\hline
\end{tabular}


rapia, ambulancias, órdenes de inyectables. d) Solicitud de incapacidad temporal (IT) o partes de confirmación de IT e) Solicitud de derivaciones a especialistas f) Solicitud de petición de pruebas complementarias g) Recogida de resultados de pruebas complementarias. D) $\mathrm{Mo}$ dalidad de consulta. En cuanto a la modalidad de consulta se consideraron los siguientes: a) Entre pacientes: cuando la consulta era solicitada al introducirse el paciente entre otros pacientes citados. b) Acompañando al paciente: cuando para la realización de la consulta se aprovecha la consulta realizada por un paciente citado. c) Al final de la consulta: visitas realizadas a pacientes que esperan la finalización de la consulta o cualquier otro momento después de ya finalizada la consulta de demanda. d) Visitas realizadas antes de iniciar la consulta de demanda. E) Momento de consulta: distinguiendo entre la jornada de mañana y la de tarde.

Por otro lado, en todos los casos, se registraba el tipo de consulta, es decir, si la consulta era realizada de forma directa o indirecta, entendiendo por consulta indirecta aquella en la que no está presente el paciente ${ }^{5}$. También se registraba quiên era la persona delegada para la realización de dicha consulta indirecta: padre, madre hijo, hija, marido, mujer, otros (amigos, compañeros de trabajo, cuidadores, otros familiares, etc.).

El estudio se llevó a cabo con el programa SPSS para Windows, versión 7.0, utilizando las pruebas descriptivas pertinentes, así como las pruebas para comparación de medias y proporciones. Para el análisis multivariante se utilizó el análisis de correspondencias simple, aplicado a una matriz de Burt, técnica que permite analizar tablas disyuntivas completas mediante el programa SPSS 4.0. Además, al objeto de verificar los resultados obtenidos y de corroborar qué categorías de las variables eran susceptibles de eliminación directa, se procedió al análisis de la información mediante el programa SPAD.N 2.5. En este caso, se realizó Análisis Factorial de Correspondencias Múltiples $^{6}$, siendo el entorno MS-DOS el marco de aplicación de ambos programas.

Como en el caso de cualquier técnica de análisis de datos multivariante, esta aproxima- ción aporta una dimensión a las técnicas de análisis bivariante utilizadas en el apartado anterior, por lo que las conclusiones que pueden ser extraídas del análisis, no sólo harán referencia a las variables tomadas de dos en dos, sino a todas conjuntamente. Como se ha indicado anteriormente, se tomaron las variables que permitieran determinar, a través de sus interrelaciones, tanto el perfil de individuo como sus motivos, etc.

\section{RESULTADOS}

En la totalidad del período de estudio, desde el 1 de enero de 1996 al 31 de diciembre de 1998, el número de consultas sin citación previa fue de 3.428 , lo que constituye el 14,19 $\%$ de la totalidad de visitas realizadas en consulta a demanda, siendo este porcentaje del 15 , 17 y $14 \%$ para 1996, 1997 y 1998 respectivamente. Se observa, además, la existencia de una relación positiva y estadísticamente significativa entre el número de visitas/día realizadas a demanda con citación previa y el número de visitas/día realizadas sin citación previa, con un coeficiente de correlación lineal de Pearson de $0,08(p=0,02)$.

\section{Análisis univariante (tabla 2):}

Durante el período de estudio del 1 de octubre de 1997 al 31 de enero de 1998, el total de visitas sin citas fue de 339 , que sobre un total de 2.435 visitas representan un $13,92 \%$, con una media de 4,35 visitas/dia $(\mathrm{DE}=2,39)$ y un rango de 0 a 11 visitas/día. Para el estudio descriptivo se utilizaron 329 visitas, desechándose 10 por faltar algunas de las variables. La edad media de los pacientes, que son objeto de consulta sin cita previa, es de 49,73 $(\mathrm{DE}=19,72)$, siendo ligeramente superior el número de mujeres frente al de hombres (un $52,9 \%$ frente a un $47,1 \%$ respectivamente). La persona que acude a la consulta sin cita previa suele ser atendida después de finalizar la consulta a demanda $(41,3 \%)$ o durante las consultas, ya que acompañan a un paciente con cita previa $(41,6 \%)$. Los principales motivos que provocan estas consultas sin cita previa son dos, la solicitud de recetas $(51,7 \%)$ y la consulta por 
Tabla 2

Descripción variables

\begin{tabular}{|c|c|c|}
\hline Variable & Frecuencia & $\%$ \\
\hline \multicolumn{3}{|l|}{ GRUPOS DE EDAD } \\
\hline Hasta 65 años & 232 & 70,51 \\
\hline 65 ó más años & 97 & 29,48 \\
\hline \multicolumn{3}{|l|}{ SEXO } \\
\hline Hombre & 155 & 47,10 \\
\hline Mujer & 174 & 52,90 \\
\hline \multicolumn{3}{|l|}{ JORNADA } \\
\hline Mañana & 276 & 83,90 \\
\hline Tarde & 53 & 16,10 \\
\hline \multicolumn{3}{|l|}{ MODO CONSULTA } \\
\hline Entre pacientes & 48 & 14,59 \\
\hline Acompañando pacientes & 137 & 41,64 \\
\hline Antes consulta & 8 & 2,43 \\
\hline Final consulta & 136 & 41,34 \\
\hline \multicolumn{3}{|l|}{ MOTIVO CONSULTA } \\
\hline Enfermedad & 104 & 31,61 \\
\hline Receta & 170 & 51,67 \\
\hline Informes & 8 & 2,43 \\
\hline IT & 28 & 8,51 \\
\hline V. derivación & 12 & 3,65 \\
\hline Solicitud Analítica & 5 & 1,52 \\
\hline Resultados & 2 & 0,61 \\
\hline \multicolumn{3}{|l|}{ TIPO DE CONSULTA } \\
\hline Directa & 179 & 54,40 \\
\hline Indirecta & 150 & 45,60 \\
\hline \multicolumn{3}{|l|}{ SEXO DE LA PERSONA } \\
\hline \multicolumn{3}{|l|}{ DELEGADA } \\
\hline Hombre & 91 & 22,67 \\
\hline Mujer & 34 & 60,67 \\
\hline \multicolumn{3}{|l|}{ PERSONA DELEGADA } \\
\hline Padre & 3 & 02,00 \\
\hline Madre & 22 & 15,00 \\
\hline Hijo & 5 & 03,00 \\
\hline Hija & 29 & 19,00 \\
\hline Mujer & 40 & 27,00 \\
\hline Marido & 26 & 17,00 \\
\hline Otros & 25 & 16,00 \\
\hline
\end{tabular}

IT= Incapacidad temporal; $\mathrm{V}$. Derivación= Volantes de derivación. enfermedad $(31,6 \%)$, existiendo un porcentaje ligeramente superior de las consultas que son realizadas de forma directa, es decir con presencia en la consulta de la persona interesada $(54,4 \%)$. Las personas delegadas para la realización de las consultas indirectas $(45,6 \%)$ son, en su mayoría, mujeres $(60,7 \%)$, ocupando las esposas de los pacientes la primera posición, seguidas de las hijas y de las madres. Sin embargo, en caso de ser varones las personas delegadas, son los maridos fundamentalmente quienes ejercen dicha función.

\section{Análisis bivariante:}

Los resultados del análisis bivariante aparecen en la tabla 3 , en la que se muestra las relaciones significativas al $95 \%$ entre las categorías de respuesta de todos los posibles cruces de variables objeto de estudio, utilizando como estadístico de contraste el residuo ajustado y considerando como valor crítico 1,96.

$\mathrm{Si}$ atendemos a la edad de las personas que han acudido a las consultas sin cita previa, el análisis de sus medias únicamente muestra diferencias estadísticamente significativas en dos de las variables analizadas, el tipo y el motivo de consulta. Mientras que, en el resto de las variables analizadas, la edad media de los individuos se sitúa entre los 49 y 50 años, en el caso de que la consulta sea directa, los individuos poseen una edad media superior (53 años) que aquellos que la realizan de forma indirecta (47 años). En cuanto al motivo de la consulta, existen diferencias significativas entre la edad media de los pacientes que acuden para la realización de partes de bajas laborales o de confirmación en las mismas ( 37 años), de los que acuden por recetas (54 años) y los que acuden por enfermedad (46 años).

En lo que respecta al sexo de los pacientes, únicamente aparecen diferencias significativas entre hombres y mujeres en las variables Motivo de consulta y Modo de la consulta. Así, las mujeres consultan en mayor proporción por solicitud de volantes de derivación y que, normalmente, suelen aprovechar la consulta realizada por otro paciente citado, mientras que los hombres consultan en mayor proporción por temas relacionados con la IT y 
Tabla 3

Descripción variables

\begin{tabular}{|c|c|c|c|c|c|c|c|c|c|c|c|c|c|c|}
\hline \multicolumn{2}{|c|}{} & \multicolumn{9}{c|}{ TIPO } & \multicolumn{7}{c|}{ MOTIVO } & \multicolumn{5}{c|}{ MODO } \\
\hline & & IND & DIR & ENF & REC & INF & IT & DER & ANA & RDO & ENT & ACO & ANT & DCO \\
\hline SEXO & H & & & & & & + & - & & & & - & & + \\
\hline M & & & & & & - & + & & & & + & & - & \\
\hline MOM & MN & - & + & & - & & + & & & & & - & - & + \\
\hline T & + & - & & + & & - & & & & & + & + & - & \\
\hline TIPO & IND & & & - & + & & - & & & & - & + & & - \\
\hline DI & & & + & - & & + & & & & + & - & & + & \\
\hline MOTV & ENF & & & & & & & & & & & - & & + \\
\hline REC & & & & & & & & & & & + & & - & \\
\hline INF & & & & & & & & & & & & & & \\
\hline IT & & & & & & & & & & & - & & + & \\
\hline DER & & & & & & & & & & & & & & \\
\hline ANA & & & & & & & & & & & & & & \\
\hline RDO & & & & & & & & & & & & & + & \\
\hline
\end{tabular}

* Se muestran únicamente los resultados significativos al $95 \%$.

El signo + indica la existencia de más personas en ese grupo que las que deberáa haber y el signo - lo contrario.

$\mathrm{IND}=$ Indirecta; $\mathrm{DIR}=$ Directa; $\mathrm{ENF}=$ Enfermedad; REC $=$ Recetas; $\mathrm{INF}=$ Informes; $\mathrm{IT}=$ Incapacidad temporal; $\mathrm{DER}=\mathrm{Derivaciones} ;$ ANA $=$ Analíticas; $\mathrm{RDO}=$ Resultados; $\mathrm{ENT}=$ Entre pacientes; $\mathrm{ACO}=\mathrm{Acompañindo} \mathrm{al} \mathrm{paciente;} \mathrm{ANT}=$ Antes de la consulta; $\mathrm{DCO}=\mathrm{Después}$ de la consulta; $\mathrm{H}=$ Varón; $\mathrm{M}=$ Mujer; $\mathrm{MOM}=$ Momento de la consulta; $\mathrm{MN}=$ Mañana; $\mathrm{T}=\mathrm{Tarde} ; \mathrm{MOT}=$ Motivo de la consulta.

utilizan el tiempo posterior a la finalización de la consulta.

Si analizamos la muestra según el horario de la jornada laboral, mañana o tarde, vemos que emergen diferencias significativas en todas las variables, a excepción, como ya hemos visto, de la variable sexo. Por las mañanas es mayor el porcentaje de las visitas directas realizadas al final de la consulta, cuyas causas principales están relacionadas con la IT, mientras que por las tardes es mayor el porcentaje de las visitas indirectas, que se producen principalmente acompañando a un paciente citado o antes de comenzar la consulta, por causas relacionadas con la solicitud de recetas.
Al estratificar por tipo de consulta (directa o indirecta) se observan diferencias significativas en lo que respecta al modo, momento y motivo de la consulta. Ya hemos comentado que las consultas directas se producen mayoritariamente por la mañana, por causas relacionadas con enfermedades y partes de IT, y que son atendidas entre pacientes o después de la consulta, produciéndose los resultados contrarios en las consultas indirectas.

Al analizar si la persona delegada, que realiza la consulta indirecta, varía en función del motivo de consulta, los únicos resultados que podemos destacar por su significación nos indican que, por motivo de enfermedad, son fundamentalmente las madres las personas 
delegadas, mientras que los hijos son quienes sustituyen a los padres cuando se trata de solicitar volantes para la derivación al especialista hospitalario o bien para la solicitud de ciertos informes $y$, por último, que cuando la consulta se refiere a un tema de índole laboral la realizan fundamentalmente otras personas próximas al paciente como amigos, compañeros de trabajo u otros familiares.

\section{Análisis multivariante}

En primer lugar, se obtuvo la matriz de Burt, que recoge las frecuencias con las que se han producido simultáneamente dos modalidades de dos cuestiones diferentes. A partir de esta información se aplicará un Análisis Factorial de Correspondencias, con el objeto de determinar las relaciones que existen entre todas las modalidades de las preguntas objetivo del análisis. En segundo lugar, de la información base, referida a las categorías de respuesta postuladas en la encuesta, cabe resaltar que algunas de ellas fueron eliminadas y otras fueron recodificadas. Esta decisión fue tomada, tanto por criterios objetivos como subjetivos. Respecto a los criterios, este tipo de eliminación puede ser llevado a cabo mediante el programa SPD.N, esto es, uno de sus outputs más importantes, permite, a través de un análisis previo, determinar aquellas categorías que a efectos del análisis posterior podrían distorsionar los resultados, debido a la escasa magnitud de la frecuencia absoluta en la categoría de respuesta en una variable. Mientras que la dimensión subjetiva se introdujo a la hora de determinar su eliminación o inclusión, tomando como referencia una categoría más amplia de la variable.

Respecto a la variable edad, se consideraron tres categorías: menores de 41 años (ED1), entre 41 y 64 años (ED2), ambas incluidas, y mayores de 64 años (ED3). En este caso, el criterio seguido fue el de establecer grupos comparables en términos absolutos que nos permitieran obtener conclusiones sustantivas. En este estudio, dada la naturaleza de las variables consideradas, se optó por la recodificación de categorías de respuesta en una de las variables de interés y de la eliminación de casos en otras, esto es, en un caso se creó una categoría de res- puesta más amplia y en otro, simplemente, se procedió a la eliminación de la categoría. Así, en la variable modalidad de consulta se consideraron tres posibles valores, siendo estos: entre pacientes (ENT), acompañando a otro paciente $(\mathrm{ACO}) \mathrm{y}$, por último, después de la consulta (DCO). Se eliminó la respuesta antes de la consulta, dada la baja frecuencia absoluta observada. En la variable motivo de consulta, de los siete valores iniciales se pasó a considerar únicamente tres: enfermedad (ENF), recetas (REC) y «papeles» (PAP), término que engloba las otra cinco categorías restantes. Por lo tanto, el número de categorías objeto a relacionar es de quince. A continuación se presentan las siglas utilizadas, de las quince categorías, a efectos de la representación gráfica:

$\mathrm{EDAD}(\mathrm{ED} 1, \mathrm{ED} 2, \mathrm{ED} 3)$

SEXO (VAR,MUJ); VAR= Varón y MUJ= Mujer

MOMENTO (MAÑ,TAR); MAÑ = Mañana y TAR= Tarde

TIPO (IND,DIR); IND= Indirecta y DIR= Directa

MODALIDAD (ENT, ACO, DCO)

MOTIVO (ENF, REC, PAP)

Si se retienen las cuatro primeras dimensiones, el porcentaje de inercia explicada supera el $70 \%$ del total, siendo el total 0,285 . Las contribuciones, en términos de inercia explica$\mathrm{da}$, de cada una de dichas dimensiones, fueron el $34,0 \%$, el $13,8 \%$, el $12 \%$ y el $11,8 \%$, respectivamente. Así pues, se optó por retener únicamente las dos primeras dimensiones, ya que con dichas dimensiones (DIM1 Y DIM2) el porcentaje de inercia explicado asciende al $47,8 \%$, esto es, prácticamente la mitad de la inercia existente. Dicha determinación se tomó teniendo presentes la parsimonia y el porcentaje de inercia explicado.

Del análisis conjunto de las contribuciones de las dimensiones a la inercia de las categorías y de las contribuciones de las categorías a la inercia de las dimensiones (tablas 4 y 5 ) se desprenden los siguientes resultados en torno a la calidad de representación de las categorías analizadas. No se puede hacer ningún comentario respecto a la categoría ENT de la variable Modalidad de consulta, dado que, en la dimensiones consideradas no queda aceptablemente 
Tabla 4

Contribuciones de las dimensiones a la inercia de cada categoría

\begin{tabular}{|llll|}
\hline & DIMI & DIM2 & TOTAL \\
\hline ED1 & 0,36 & 0,00 & 0,36 \\
ED2 & 0,11 & 0,10 & 0,21 \\
ED3 & 0,11 & 0,09 & 0,20 \\
VAR & 0,02 & 0,41 & 0,43 \\
MUJ & 0,02 & 0,41 & 0,43 \\
MAN & 0,29 & 0,13 & 0,42 \\
TAR & 0,29 & 0,13 & 0,42 \\
IND & 0,68 & 0,06 & 0,74 \\
DIR & 0,68 & 0,06 & 0,74 \\
ENT & 0,01 & 0,01 & 0,02 \\
ACO & 0,66 & 0,02 & 0,68 \\
DCO & 0,59 & 0,04 & 0,63 \\
ENF & 0,40 & 0,38 & 0,78 \\
REC & 0,73 & 0,03 & 0,76 \\
PAP & 0,19 & 0,30 & 0,48 \\
\hline
\end{tabular}

DIM1 = Dimension 1; DIM2= Dimensión 2;ED1 = Menores de 41 años; ED2= Entre 41 y 64 años, ambos incluidos; ED3 = mayores de 64 años; VAR = Varón; $M U J=$ Mujer; $\mathrm{MN}=$ Mañana; TAR= Tarde $; \mathrm{IND}=$ Indirecta $; \mathrm{DIR}=$ Directa; $\mathrm{ENT}=$ Entre pacientes; $\mathrm{ACO}=$ Acompañando al paciente; $\mathrm{DCO}=$ Después de la consulta; $\mathrm{ENF}=$ Enfermedad $\mathrm{REC}=$ Recetas; PAP $=$ Papeles.

representada. Esta categoría define prácticamente la cuarta dimensión, la cual no es considerada en este análisis. Aunque las categorías MAÑ, ED1, ED2 Y ED3 presentan unas bajas contribuciones de inercia respecto a las dos dimensiones consideradas, no cabe su eliminación, ya que quedan explicadas con porcentajes superiores al $20 \%$, superando incluso MAÑ el $40 \%$. Respecto a las categorías restantes, mediante las dos dimensiones retenidas, se explican más del $50 \%$ de IND, DIR, ACO, DCO, ENF y REC, mientras que se supera el $40 \%$ en VAR, MUJ, TAR y PAP. Por tanto, únicamente se elimina del análisis la categoría ENT. Así pues, se pasa a analizar la posición que ocupan en los ejes cada una de ellas (figura 1).
Tabla 5

Contribuciones de las categorias a la inercia de cada dimensión

\begin{tabular}{|lll|}
\hline & DIMI & DIM2 \\
\hline ED1 & 0,07 & 0,00 \\
ED2 & 0,02 & 0,05 \\
ED3 & 0,02 & 0,04 \\
VAR & 0,00 & 0,16 \\
MUJ & 0,00 & 0,14 \\
MAN & 0,02 & 0,02 \\
TAR & 0,08 & 0,08 \\
IND & 0,14 & 0,03 \\
DIR & 0,12 & 0,03 \\
ENT & 0,00 & 0,01 \\
ACO & 0,14 & 0,01 \\
DCO & 0,12 & 0,02 \\
ENF & 0,09 & 0,22 \\
REC & 0,13 & 0,01 \\
PAP & 0,05 & 0,19 \\
\hline
\end{tabular}

DIM1 = Dimensión $1 ;$ DIM2 $=$ Dimensión 2; EDI $=$ Menores de 41 años; ED2= Entre 41 y 64 años, ambos incluidos; $E D 3=$ mayores de 64 años; $V A R=$ Varón; $M U J=$ Mujer; $\mathrm{MN}=$ Mañana; $T A R=$ Tarde $; \mathrm{IND}=$ Indirecta; $\mathrm{DIR}=$ Directa; $\mathrm{ENT}=$ Entre pacientes; $\mathrm{ACO}=$ Acompañando al paciente; $\mathrm{DCO}=$ Después de la consulta; $\mathrm{ENF}=$ Enfermedad; $\mathrm{REC}=$ Recetas; $P A P=$ Papeles.

Las categorias que nos permiten definir la primera dimensión (DIM1) son, en la parte negativa del eje representativo, ENF, DIR, ED1, DCO y PAP y en la positiva TAR, ACO, REC e IND. Por ello, cabe concluir que: las consultas cuyo motivo es enfermedad o «papeles» suelen ser realizadas de manera directa y después de la finalización de la consulta. Además, los individuos que realizan este tipo de consultas suelen tener menos de 41 años. Aquellos pacientes que acostumbran a ir por la tarde, sin cita previa, suelen hacerlo con el objetivo de conseguir recetas $y$ suelen ser consultas indirectas realizadas por otros pacientes que consultaban con citación previa. 
Figura 1

Representación de las categorías analizadas en las dos primeras dimensiones

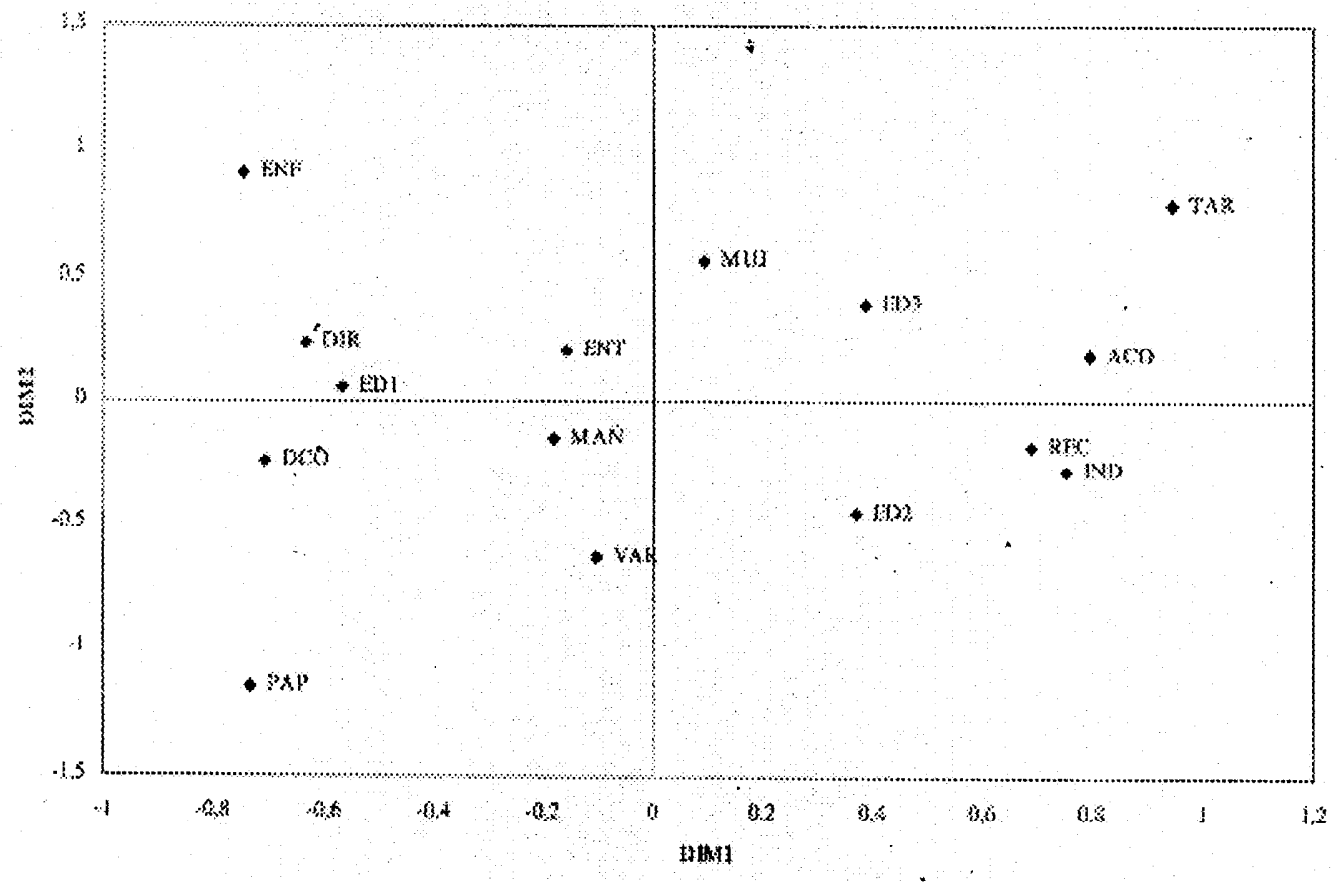

Cinco son las categorías recogidas en la segunda dimensión (DIM2): VAR, MUJ, ENF, PAP y TAR. En la parte positiva del eje se sitúan las categorías MUJ, ENF y TAR, y en el eje negativo VAR y PAP. Así, se observa que este eje posiciona la variable sexo (MUJ versus VAR) y permite distinguir entre dos de los motivos de consulta (ENF versus PAP). En cierta forma añadc contenido a lo comentado anteriormente, sólo con la dimensión 1.

Para poder realizar algún comentario, en relación a las categorías ED2, ED3 y MAÑ, es necesario utilizar las dos dimensiones dado que, aún mostrando bajas contribuciones $y$, consecuentemente, estar en la figura 1 cerca del origen de coordenadas, se ha considerado pertinente su inclusión (el porcentaje de inercia explicado de la categoría MAÑ asciende a un $42 \%$, de la categoría ED2 $21 \%$ y de la ED3 $20 \%$ ), aunque asumiendo las limitaciones que existen al quedar representadas tan cerca del origen de coordenadas. De la observación del gráfico en cuadrantes se desprenden los siguientes comentarios:

- G1: Primer cuadrante: D1M1+ y D1M2 + : Las mujeres mayores de 64 años suelen ser un colectivo que aprovecha que se encuentra en el consultorio acompañando a otras personas por la tarde.

- G2: Segundo cuadrante: D1M1+y D1M2-: Existe un colectivo cuya edad está comprendida entre 41 y 64 años que acude al médico sin cita previa al objeto de demandarle recetas que no son para ellos.

- G3: Tercer cuadrante: D1M1- y D1M2-: Los individuos que no tienen cita previa y que acuden al médico después del horario de consulta son mayoritariamente hombres y su motivo suele ser la petición de volantes, analíticas, bajas, etc. y este hecho suele ser habitualmente por las mañanas. 
- G4: Cuarto cuadrante: D1M1- y D1M2+: Existe un colectivo, frecuentemente no son personas mayores, que optan por acudir personalmente al médico cuando están enfermos, aún cuando no tienen cita previa.

- G1 y G2: si el análisis no se realiza estrictamente atendiendo a los cuadrantes respecto de la dimensión 1 , tendríamos, además, tres aspectos a destacar (REC, IND, ED2) respecto de motivo, a quién va dirigida la consulta y una ampliación del intervalo de edad. Esto es, le añadiríamos que son consultas indirectas con motivo de solicitar recetas.

\section{DISCUSION}

La presencia de un $15 \%$ de consultas, realizadas a pacientes que no tienen asignado un tiempo específico parece alto, máxime teniendo en cuenta la existencia, dentro del organigrama de funcionamiento del centro, de dos médicos, cada día, dedicados a la atención del paciente sin citación. La alta frecuencia de motivos burocráticos, la prescripción de tratamientos repetidos ${ }^{12}$, así como la continuidad en los cuidados como indicador de satisfacción $^{13,14}$, podría justificar dicha actitud. La presencia de una relación positiva entre el número de visitas/día realizadas sin citación previa y el de las visitas/día con citación podría explicarse por la circunstancia de que sean las visitas que se producen acompañando al paciente citado una de las formas más habituales de consultar sin cilación. El porcentaje de consultas directas e indirectas es similar, y están muy por encima del $18 \%$ observado en el conjunto de las visitas de la consulta de demanda, aparecido en otro estudio ${ }^{15}$. Estas, son efectuadas por pacientes con edades comprendidas entre 15 y 64 años, seguido por los de 65 años ó más. Los motivos por los que se realizan estas consultas indirectas son la realización de recetas, mientras que la consulta con el paciente presente tiene como motivo un problema de salud. Es notable, más desde una perspectiva cualitativa que cuantitativa, que el $14 \%$ de las consultas indirectas tengan como motivación la presencia de un proceso patológico, con lo que ello supone de pérdida de relación médicopaciente, así como de una adecuada valoración del proceso que motiva la consulta. Las mujeres son las que con mayor frecuencia hacen de portavoz del paciente ausente, ya que tradicionalmente vienen asumiendo el papel de cuidadoras 16, además de que realizan menos frecuentemente un trabajo remunerado $\mathrm{y}$, por tanto, tienen más disponibilidad horaria. En conjunto, se identifican dos perfiles de utilizadores de la consulta sin citación previa: los pacientes jóvenes que consultan por enfermedad o tareas burocráticas al finalizar la consulta de mañana, y aquellos que ocasionan consultas indirectas, realizadas por la tarde y llevadas a cabo por personas que consultan por ellas mismas y con citación previa, cuyo objetivo es la solicitud de recetas. En el primero de los casos se podría identificar un defecto de accesibilidad, ya que podría tratarse de pacientes que aprovechan el período de mediodía, entre la jornada laboral de mañana y tarde, lo que implicaría una limitación respecto a la accesibilidad que debería proporcionar la «jornada deslizante» $o$, igualmente, el inicio de la consulta a las 9 horas, con finalización a las 11,45 , justificaría el mayor número de visitas al finalizar la consulta y la baja frecuencia de visitas previas al inicio de la misma, por lo que habría que esperar la inversión de las frecuencias en aquellas consultas que se inician a las 12 horas y que terminan a las 14,30 horas. La realización de posteriores estudios nos podría ayudar a aclarar estos extremos. Si se detectara una baja accesibilidad en estos pacientes, habilitar un tiempo de consulta para resolver pequeños problemas de salud o burocráticos a determinadas horas, compatibles con su jornada laboral, podría paliar este problema.

El tiempo de consulta asignado a una persona debe dedicarse exclusivamente para la misma ya que, difícilmente, podemos dedicarlo a la asistencia de otras sin que ello repercuta negativamente en los tiempos de espera. En el segundo grupo caracterizado de pacientes, se trataría de consultas indirectas para solicitar recetas, aprovechando la consulta realizada, el típico «ya que estoy aquí», consultas que, de producirse de un modo reiterado, alargan las consultas con citación más allá de lo deseable, acumulando minutos de retraso, con la consi- 
guiente desesperación del médico y de la persona que espera, que va viendo alejarse su hora de citación. Este colectivo debería de cambiar de hábitos, previa concienciación de que están generando al resto de pacientes un problema.

Para la correcta organización de los tiempos de consulta proponemos como medidas correctoras deseables las siguientes:

- Informar a la población de la necesidad de realizar la citación de todos aquellos pacientes que sean objeto de consulta, estén presentes o no e independientemente del motivo, para adecuar el número de personas visitadas al de registradas en la hoja de citación que, por un lado falsean los datos, no permitiendo conocer la realidad de la demanda y que, por otro lado, ocasionan un aumento del tiempo de espera de los pacientes citados, al utilizar el destinado a un solo paciente para la visita de otros no citados

- Concienciar a los usuarios para que soliciten los fármacos contenidos en las cartillas para prescripciones repetidas mediante la utilización de la organización existente para ello.

- Disuadir sobre la utilización de la modalidad de consulta indirecta, sobre todo cuando ello implique el tratamiento de determinados procesos que, en ocasiones, son considerados banales por el propio paciente o sus familiares, sin una consulta previa que permita la valoración adecuada por parte del profesional sanitario.

- Implicar al personal de admisión en fomentar la citación adecuada a la consulta, conteniendo en ocasiones una accesibilidad excesiva que interfiere en perjuicio de otros pacientes y de otras actividades, tanto asistenciales como de otra índole, y que tienen que ser postpuestas o incluso aplazadas.

- Establecer, de confirmarse en posteriores estudios la existencia de grupos de población con problemas de accesibilidad, medidas organizativas más eficaces que las actuales que permitan paliar este problema, sin menoscabar la atención al resto de los pacientes o el desarrollo de otras actividades del equipo de atención primaria.

\section{AGRADECIMIENTOS}

Agradecemos al profesor Ildefonso Grande, profesor de la Universidad Pública de Navarra, sus comentarios, así como que nos permitiera utilizar el programa SPAD-N 2.5. Agradecemos al Dr. Mariano Rodríguez Torrente, Coordinador de la Unidad Docente de Huesca los comentarios realizados.

\section{BIBLIOGRAFIA}

1. Penchansky R, Thomas JW. The concept of access: Definition and relationship to consumer satisfaction. Med Care 1981; 19: 127-140.

2. Martín Zurro A, Huguet M y García Cardona F. La organización de las actividades en Atención Primaria. En: Martín Zurro A y Cano Pérez JF. Atención Primaria. Conceptos, organización y práctica clínica, $3^{\mathbf{a}}$ ed. Madrid: Doyma Libros SA. 1994.p. 37-38.

3. Simó Miñana J, Gálvez Candel J, Morote Sanchís MV y Palazón Ferrer G. El médico de cabecera o la búsqueda de la identidad perdida: información para internistas y otros especialis tas. Med Clin (Barc) 1997; 109: 343-348.

4. Informe económico del Programa de Cita Previa. Bilbao: Servicio Vasco de Salud; 1988

5. WONCA. Glosario Internacional de Atención Primaria. En: WONCA. Clasificaciones de la WONCA en Atención Primaria. Barcelona: Masson;1990.p. 252-268.

6. El análisis multivariante de la información (I). Los métodos de interdependencia. En: Ildefonso Grande Esteban y Elena Abascal Fernández. Fundamentos y técnicas de investigación comercial, $3^{a}$ ed. Pozuelo de Alarcón (Madrid): Esic editorial;1996.p. 486-536.

7. Haynes RM. Inequalities in health and health care service use: cvidence from the Gcncral Household Survey. Soc Sci Med 1991; 33: 361-368.

8. Zuzuárregui Gironés $\mathrm{M}$, Montesinos Alonso A, Sánchez Sánchez C, Tejedor Infantes C, 
López Jiménez J.M. Opinión de los usuarios sobre accesibilidad y atención sanitaria en un área de salud. Centro de Salud 1997; 5: 257. 262.

9. Encuesta de satisfacción de los usuarios con los servicios de Atención Primaria. Madrid: Insalud; 1997.

10. Moreno E, Gómez-Calcerrada D, Sandoval C, Cánovas J, Ramos C, Peñalver F:J, Guillén F y Marset P. Influencia de la cita previa en las consultas de atención primaria en la región de Murcia. Aten Primaria 1992; 9: 38-41.

11. Repercusiones del programa de cita previa en atención primaria de salud. Atención Primaria 1992; 10: 757-758.

12. Sáenz González MC, Mirón Canelo JA, González Celador R. Valoración de la demanda y utilización de los servicios sanitarios por parte de la población rural de la provincia de Salamanca. Encuesta por entrevista personal. Rev San Hig Publica 1991; 65: 61-70.

13. Weiss GL. Patient satisfaction with primary medical care. Evaluation of sociodemographic and predispositional factors. Med Care 1988; 26: 383-92.

14. Bernhart $\mathrm{MH}$, Wiadnyana IG, Wihardjo $\mathrm{H}$, Pohan I. Patient satisfaction in developing countries. Soc Sci Med 1999; 48: 989-96.

15. López-Miras A, Pastor Sánchez R, Pérez Fernández M y Gervas J. Consulta por terceras personas en medicina general. Aten Primaria 1994; 13: 475-479.

16. Puig C, Hernández-Monsalve LM, Gervás JJ. Los enfermos crónicos recluidos en el domicilio y su repercusión en la familia: una investigación cualitativa. Aten Primaria 1992; 10: 550-552. 